\title{
ASSESSMENT OF DIVERSITY IN SOME WHEAT GENOTYPES, BASED ON RGA MARKERS ANALYSIS
}

\author{
Bivol Ina, Gore A., Rotari Silvia \\ Institute of Genetics, Physiology and Plant Protection, Chisinau, Republic of Moldova \\ e-mail: bivolinga@yahoo.com
}

During the coevolution of plant-pathogen interactions the plants, have developed the sophisticated multi-layered immune defense systems, which involving many resistance genes (R) and their products. Most of the known R-genes and resistance gene analogs (RGAs) discovered in plants possess conserved functional domains and structural features, and can be grouped into five classes: nucleotide binding site - leucine-rich repeat (NBS-LRR), intracellular serine - threonine protein kinase (PK), leucine-rich repeat - single transmembrane region (LRR-TM), PK-LRR-TM, and signaling anchor - coiled-coil domain (SA-CC). Specific genomic DNA sequences amplified by degenerate primers based on these motifs are known as RGAs or potential R-genes, using that possible to identify and isolate of numerous related sequences of R-genes and RGAs in other plant species. Many studies have demonstrated the high level of RGA polymorphisms that may result from various mutations types such as deletions, duplications, unequal crossing-over, frame shifts due to continuous response to diverse plant pathogen populations. The RGAs technique is important tool for the development of molecular markers that are utilized for genetic diversity analysis, tagging disease resistance related traits, discovering candidate genes in several crops, and also understanding of the molecular mechanisms of R-gene function and evolution, thus enhancing numerous disease resistance by breeding programs.

The purpose of the present study was to assess the existing genetic diversity and relationships based upon several potential candidate resistance genes among some wheat genotypes.

Genetic diversity and relationship between 11 common wheat (Triticum aestivum L.) and durum wheat (T. turgidum var. durum) genotypes from the collection of the Institute of Genetics, Physiology and Plant Protection were studied by means of RGA markers. Six pairs of RGA primers (NLRR for/rev, CLRR for/rev, XLRR for/rev, RLRR for/rev, Ptokin1/2 and Ptokin3/4) derived from PK and LRR domains of R-genes were used. In RGA analysis two effective primers (XLRR for/rev and Ptokin1/2) were selected and, in consequence, RGA amplified fragments were produced in sizes ranging from 153 to $1402 \mathrm{bp}$. The number of amplicons generated by the primers ranged from 11 to 14. It was discovered that RGA primers produced 16 specific and 9 polymorphic fragments among wheat accessions. The level of polymorphism detected with primers XLRR for/rev and Ptokin1/2 was 42.86 and $27.27 \%$, respectively, that indicating the genetic variation among the examined genotypes. Genetic similarity between accessions was estimated by means of cluster analysis using Nearest Neighbor method, Euclidean distances. According to this data, the genotypes were divided into 2 distinct clusters. The first cluster was formed from two separate subclusters: subcluster 1 contained cultivars ATHLON and Lăutar; cultivars Moldova 77, Moldova 79, Moldova 5, Moldova 614, Kuialnic, Epoha and Hordeiforme 335 were clustered in subcluster 2, moreover, Kuialnic and Epoha showed a high degree of similarity. While in the second cluster there were Moldova 66 and Moldova 11.

In conclusion, RGA markers are suitable for both assessment genetic diversity and for recognition of candidate genes and also for inferring genetic relationships among wheat genotypes. Whereas, it was detected the medium level of polymorphism with 2 RGA primers, for the increase exactness scanning of the genome need to use more number of the primer combinations. This information will be highly relevant for identify and deployment of disease resistance genes with goal of development resistant genotypes of wheat to a wide array of pathogens. 